\title{
De las Asociaciones del PSA a la Cooperativa del MTE: dos décadas de activismo y participación de las mujeres en el Oeste pampeano
}

\author{
Leticia Nora García ${ }^{1}$ \\ Universidad Nacional de La Pampa \\ @ [ leticia.garcia092@gmail.com ]
}

RECIBIDO 29-06-2021

ACEPTADO 19-08-2021

Cita sugerida: García, L. N. (2021). De las Asociaciones del PSA a la Cooperativa del MTE: dos décadas de activismo y participación de las mujeres en el Oeste pampeano. Revista Huellas, Volumen 25, № 2, Instituto de Geografía, EdUNLPam: Santa Rosa. Recuperado a partir de: http://cerac.unlpam.edu.ar/ index.php/huellas

DOI: http://dx.doi.org/10.19137/huellas-2021-2526

\begin{abstract}
Resumen
Las perspectivas de Género(s) y Derechos en las Políticas Públicas pasaron de ser enunciados a implicarse lenta, recortada y paulatinamente en acciones. Las mismas perspectivas encuentran intersticios tanto en las Geografías como en los territorios, aunque aún adeudan inclusiones. En este sentido pretendemos dar cuenta de las estrategias que despliegan mujeres crianceras del oeste pampeano vinculadas con experiencias de participación y, los alcances de las políticas públicas vinculadas con sus vidas cotidianas. Al concebir a las Políticas Públicas como procesos dinámicos donde interactúan relaciones sociales y estatales, entendemos que los territorios se nutren de las mismas, particularizan problemas según los contextos y también se vuelven condicionantes, reactivos y/o reproductores de cambios y también de desigualdades. En este artículo recurrimos a dos acciones de políticas del Estado Nacional desarrolladas entre el año 2006 y la actualidad. La primera de ellas considerada directa, fue la experiencia asociativa y de participación de las mujeres en el contexto del Programa Social Agropecuario (PSA) y otra indirecta vinculada con una organización de productores y productoras coordinada por la cooperativa La Comunitaria (MTE).
\end{abstract}

Palabras clave: asociaciones; cooperativa; políticas públicas; geografías feministas

Huellas vol. 25 (2) I ISSN: 0329-0573 (impresa) / 2362-5643 (en línea) 


\title{
From PSA Associations to MTE Cooperatives: two decades of activism and participation of women in Western La Pampa
}

\begin{abstract}
Genre and Rights perspectives in Public Policies moved from the field of statements to be slowly, tailored and gradually implied in actions. These perspectives find an interstice both among geographies and territories, even though there may still be some inclusions left. In this sense, the aim of this work is to shed some light on the strategies applied by nomadic shepherds in Western La Pampa in relation to active participation experiences and on the scope of the public policies in relation to their everyday lives. Understanding Public Policies as dynamic processes where social and state relationship interact with each other lead to see territories as nourished by them, where problems are particular of different contexts and become determinant to react against and/or reproduce changes and inequalities as well. This article resorts to two National State policies developed from 2006 up to present days. The first of those actions, considered a direct one, was the associative and participative experience of women in the context of the Programa Social Agropecuario (PSA) while the other is and indirect action linked to an organization of producers who were coordinated by the cooperative La Comunitaria (MTE).
\end{abstract}

Keywords: associations; cooperatives; public policies; feminist geographies

\section{Das Associações do PSA à Cooperativa do MTE: duas décadas de ativismo e participação das mulheres no Oeste pampiano}

\begin{abstract}
Resumo
As perspectivas de Gênero (s) e Direitos nas Políticas Públicas passaram de ser enunciados a participar lenta, recortada e paulatinamente em ações. Tais perspectivas encontram interstícios tanto nas geografias quanto nos territórios. Neste sentido pretendemos explicar sobre as estratégias que realizam mulheres crianceras do Oeste pampiano vinculadas com experiências de participação e os alcances de políticas públicas vinculadas com o seu cotidiano. Ao conceber as políticas públicas como processos dinâmicos onde interatuam relações sociais e estaduais entendemos que os territórios se nutrem daquelas, naturalizam problemas segundo os contextos e também se tornam condicionantes, reativos e reprodutores de mudanças e também de desigualdades. Neste artigo apelamos a duas ações de políticas do Estado Nacional desenvolvidas entre 2006 e a atualidade. A primeira delas, considerada direta, foi a experiência associativa e de participação das mulheres no contexto do Programa Social Agropecuário (PSA) e a outra indireta vinculada com a organização de produtores e produtoras coordenada pela Cooperativa La Comunitária (TEM).
\end{abstract}

Palavras-chave: Associações; Cooperativa; Políticas Públicas; Geografias Feministas 


\section{Introducción ${ }^{2}$}

Los territorios del Oeste pampeano comparten historias comunes de despojo ambiental y social, de construcción de imaginarios alrededor del "desierto", de lo improductivo, y con ello, de variadas intervenciones del Estado, en su mayoría pensadas desde el "desarrollo" productivo extensivo pampeano. Las relaciones de género(s) desiguales se evidencian claramente, en la invisibilidad de enunciación que tienen las unidades de producción: se nombra generalmente a los puesteros, crianceros, pequeños productores, mientras las mujeres, desarrollan múltiples estrategias además de ser visiblemente las garantes de la producción caprina y reproducción de la familia. Alejandra de Arce (2020) reconoce para el contexto rural nacional que esta histórica asignación de tareas provoca, entre otras cosas, la responsabilidad exclusiva del cuidado, la diferencia en el acceso a los recursos naturales y económicos y excluye a las mujeres del acceso a la renta monetaria, de la gestión de las actividades productivas y de los espacios públicos de decisión. Traza una frontera invisible -y muchas veces infranqueable- entre los géneros (de Arce, 2020, p. 2).

A continuación, precisamos algunas consideraciones generales desde dónde abordamos el problema y seguidamente enunciamos los apartados del artículo. En este contexto consideramos que estos territorios presentan doble condición de lo que denominamos "borde": territorial y de género(s) ${ }^{3}$. El concepto de borde que sustentamos disputa "la marginalidad" de estos espacios concebidos desde un modelo de desarrollo que entiende que los mismos no se adaptan a las exigencias del mercado. Lo identificamos como espacio subalternizado en cuanto a lo territorial y a las relaciones de género(s), pero al mismo tiempo donde se disputan relaciones y por lo tanto se construyen permanentemente identidades. Comerci (2018) advierte que en el margen coexisten distintos modelos y, de este modo, constituye un intersticio para la generación de formas otras de organización espacial. El concepto de Género(s) en plural marca su desvinculación con un sexo fijo ante la multiplicidad de posibilidades de género-sexo-práctica sexual-deseo. En este sentido si bien seguimos usando el concepto de género para integrar distintas y desiguales construcciones políticas, sociales, culturales, es necesario ajustar su alcance y no acotarlo al binarismo sino a la amplitud de reconocimiento y autopercepción. En Geografía esta categoría posicionó el problema de invisibilidad de las mujeres y las desigualdades en el espacio. En 1990, Liz Bondi marcó la distinción entre Geografía Feminista y Geografía del Género. No bastaba la inclusión de la categoría como dimensión de la vida social sino la transformación cultural de las desigualdades. Los feminismos impulsan esas transformaciones: en las formas de producir conocimiento, en las formas en que vivimos y 
nos relacionamos. Decimos que lo que define a las Geografías Feministas es su praxis y la lucha por la igualdad de derechos. Desde esta perspectiva en este artículo nos centramos en recuperar experiencias de mujeres crianceras y pequeñas productoras ${ }^{4}$ incluidas en experiencias asociativas que aportan sentidos de integralidad a las políticas públicas vigentes. Es un análisis desde el territorio donde el Estado es parte de sus dimensiones constitutivas. En el camino de pensar a la Administración Pública y las políticas del Estado como procesos políticos que incluyen a quienes se dirigen las acciones, y también a quienes diseñan y las ejecutan, advertimos que los mismos no están libres de valores y que quienes intervienen son actores políticos. El trabajo tiene como objetivo relevar experiencias particulares de participación de las mujeres e identificar el impacto y los obstáculos que tuvo el Programa Social Agropecuario ${ }^{5}$ (PSA de ahora en adelante) como política pública ${ }^{6}$ que incluyó a las pequeñas productoras y productores de la agricultura familiar y el paso a otro espacio de participación y representación a partir del año 2017 vinculado al Movimiento de Trabajadores Excluidos (MTE de ahora en adelante).

Seleccionamos dos momentos vinculados con políticas públicas que favorecieron vínculos y acciones entre la población productora del Oeste y agentes promotores. El primero, entre los años 2006 y 2013 y, el segundo, desde 2017 a la actualidad. Cabe mencionar que se incluye la conformación de la Cooperativa La Comunitaria (MTE) ${ }^{7}$ en el año 2017 como un proceso autogestivo promovido por otros movimientos cooperativos extra locales y sociales vinculados a la Economía Popular. El reconocimiento por parte del Estado de la categoría de trabajadores y trabajadoras de la Economía Popular convierte esta acción en una política pública entendiéndola según Lattuada, Nogueira y Urcola (2015), a la toma de decisiones sobre un problema definido. El cambio de gestión de gobierno en Argentina, en 2019 redefine criterios en la organización de la Secretaria de Agricultura Familiar Campesina e Indígena (SAFCl) entre los cuales, los movimientos sociales ingresan en los programas y acciones. El MTE (vinculado con la Cooperativa de productoras y productoras analizada en este estudio) integra el equipo de la SAFCI sede La Pampa, junto a otros movimientos. Presentamos inicialmente los aspectos metodológicos y describimos el territorio objeto de análisis. Le siguen consideraciones del PSA y su implementación en la provincia, los casos de mujeres presidentas de Asociaciones en el contexto del mismo Programa y las distancias entre formulación e implementación del mismo. Desde esta complejidad, focalizamos en el caso del PSA, el lugar de reconocimiento de las mujeres por parte del programa y desde los agentes que llevaron adelante las acciones en territorio, también por parte de las unidades familiares, y de las propias mujeres que lideraron y lideran los grupos. 
En el mismo sentido analizamos el surgimiento y las relaciones al interior de la Cooperativa La Comunitaria de Santa Isabel y las que establecen las productoras con acciones de la reciente SAFCI, junto a los impactos y dinámicas del ejercicio asociativo y el reconocimiento de ser trabajadoras de la Economía Popular. Consideramos que las relaciones que se establecen entre actores, organismos y organizaciones en cada territorio contienen marcas socioculturales particulares, a la vez que construyen nuevas territorialidades o control del espacio.

\section{Consideraciones metodológicas}

Optamos por una metodología cualitativa a partir de la cual se recuperan entrevistas en profundidad realizadas a mujeres y familias en los puestos desde el año $2006^{8}$. En particular en este trabajo se mencionan las voces de dos ex presidentas de asociaciones, la actual presidenta de la cooperativa y crianceras que participaron y participan en ambas experiencias. También se cita a extensionistas y responsables del PSA, y al coordinador de la Zona 1 Oeste de la SAFCl. Como fuentes secundarias recurrimos a datos estadísticos nacionales y provinciales, información en línea de los programas, y leyes disponibles en lugares oficiales del Estado (Argentina.gob) y la publicación regional Ecos del Oeste-Ecos de la Región, de la Subsecretaría de Desarrollo Rural y Agricultura familiar, Delegación La Pampa. Si bien el problema nace a partir de reconocer la persistencia de condiciones de exclusión territorial en el tiempo a pesar de las actuaciones del Estado en el Oeste pampeano, ese contexto sitúa a las mujeres como protagonistas centrales que resuelven la vida cotidiana en los puestos como prácticas instituidas culturalmente. La lectura desde la perspectiva de las Geografías Feministas propone una pluralidad de acercamientos a la producción de conocimientos. Al respecto Sofía Zaragocín propone que hay que "jugar con la construcción de conocimiento geográfico. Parte de eso, es poner mayor importancia en el método y no en la teoría. Cómo hacemos el feminismo descolonial ${ }^{9}$ latinoamericano, es más importante que nuestra teoría lo sea. (Zaragocín, 2020, p 27). Entonces, el hacer geografía desde los feminismos situados en territorios implica:

- Poner en cuestión los presupuestos contenidos en políticas y programas que consoliden exclusiones, estereotipos y lugares según género $(\mathrm{s})$.

- Otorgar relevancia a los intereses y perspectivas de las personas autodefinidas mujeres vinculadas con las políticas estatales en el territorio. 
- Superar las aproximaciones evaluativas, tanto de una política pública como de las organizaciones populares y pensarlas en clave de integralidad, de derechos, anclada en políticas y epistemologías descoloniales.

- Incluir los sentidos y las emociones en el análisis y comprensión de sus realidades

- Reconocer las diferencias que tenemos las mujeres al acceso a los derechos.

- Instituir a las mujeres como productoras de conocimiento.

Para aproximar el análisis integral de las políticas públicas en el territorio desde perspectivas feministas recuperamos a Pecheny y Palumbo (2017) quienes plantean la escena en tanto relaciones sociales que en ocasiones pasan desapercibidas, pero que son fuente donde se amalgaman representaciones, se incluyen sentidos e interacciones.

\section{Situarnos en el territorio}

El área de influencia de ambas organizaciones incluye a pequeñas productoras y productores localizados y distribuidos en dos ejidos municipales: Santa Isabel y Limay Mahuida de la Provincia de La Pampa, Argentina (Figura $\mathrm{N}^{\circ} 1$ ).

En la (Figura $\mathrm{N}^{\circ} 1$ ) con referencia color: azul y verde, se localiza el área de influencia de las dos Asociaciones El Salitral y El Paso seleccionada para el análisis. En la misma figura se superpone un área sombreada de norte a sur que aproxima la localización de productores/as asociados/as actualmente a la Cooperativa La Comunitaria de Santa Isabel quien conforma la rama rural del Movimiento de Trabajadores Excluidos (MTE) de la Confederación de los trabajadores de la Economía Popular (CTEP).

Advertimos que generalmente se referencia al Oeste pampeano con atributos generales aunque esa "generalidad" encierra distintas actividades productivas como la ganadería extensiva, agricultura bajo riego; diferentes modos de vida vinculados a grupos de población auto referenciada "criolla" y comunidades originarias; entre muchas otras dimensiones a incluir. Los centros urbanos cabeceras de los departamentos distan entre 300 y $350 \mathrm{~km}$ a la ciudad capital. Las características ambientales áridas y semiáridas se asocian a vegetación de monte ralo y variedad de geo formas modeladas por la erosión en un ambiente definido según Covas (1998); Comerci (2014), como depresión fluvial de la cuenca Atuel-DesaguaderoSalado, con escurrimiento intermitente por usos en la cuenca alta y media. La población dispersa está distribuida en puestos que pueden apreciarse en los puntos cartografiados en la Figura $N^{0} 1$. La comunicación terrestre 
es a través de picadas (contrafuegos) y caminos vecinales de tierra que no siempre se encuentran transitables. La Ruta Nacional No 151 conecta el Norte y Sur del Oeste provincial y con la Provincia de Mendoza y Río Negro.

Figura $N^{\circ}$ 1: Localización y áreas de influencia de las Asociaciones El Salitral y El Paso (PSA) y Cooperativa La Comunitaria

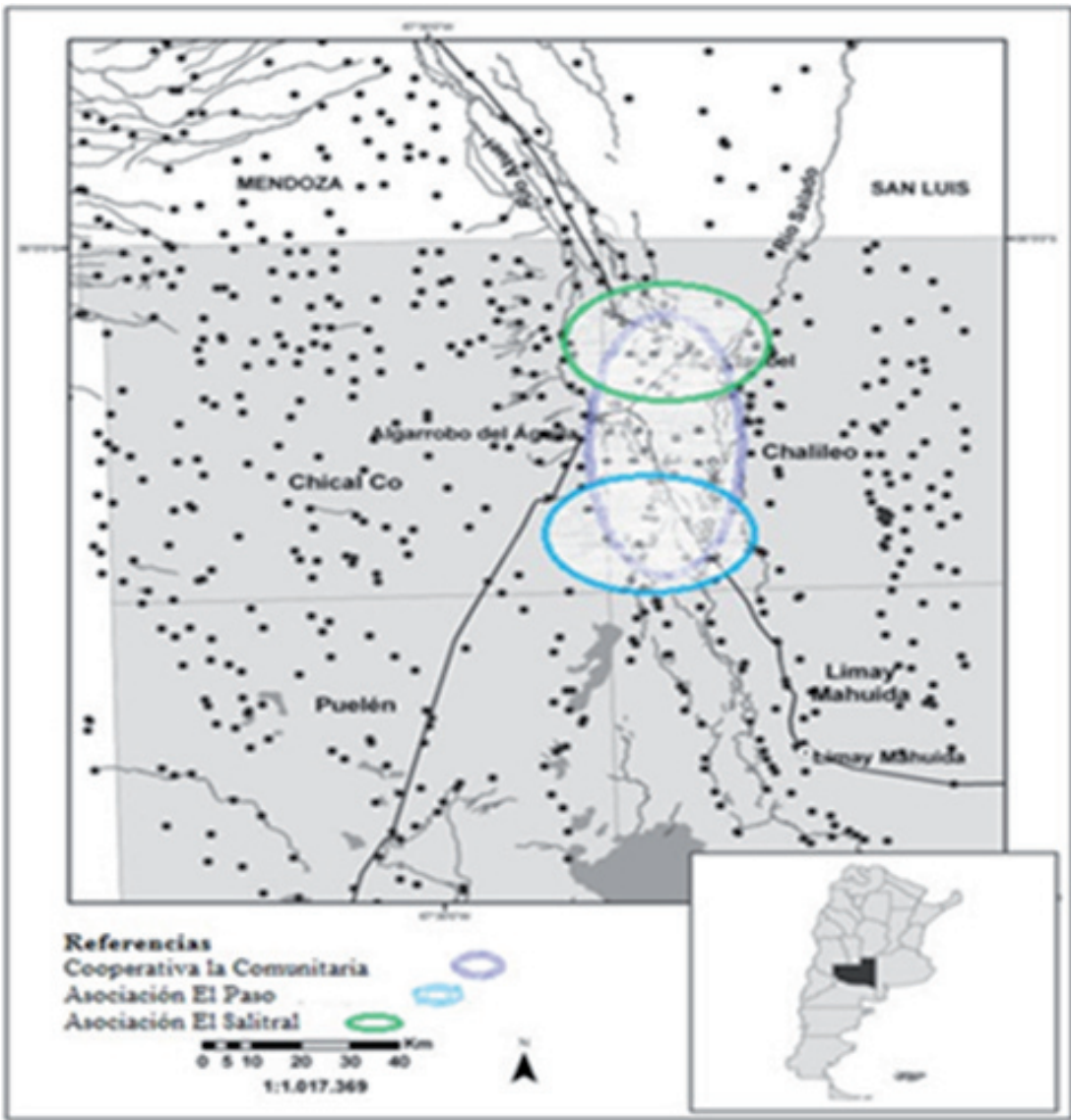

Fuente: Elaboración propia en base a Pombo, D Atlas Geográfico y Satelital de la provincia de La Pampa, 2014

El Censo Nacional 2010 registró 516 habitantes en el Departamento Limay Mahuida y 3051 habitantes en el Departamento Chalileo. Los dos ejidos mencionados reúnen la mayoría de habitantes de los Departamentos. Discriminar si es población rural o urbana resulta un tanto difuso ya que en muchos casos existe la doble residencia. Pero es interesante des- 
tacar que cuando inicia el PSA el porcentaje de población relevada como rural era del $25 \%$ en el Dpto. Chalileo y $75 \%$ en el Dpto. Limay Mahuida, según Censo Nacional 2001. Las características demográficas del espacio estudiado vinculan la intensidad de las relaciones entre vulnerabilidad y pobreza. Las condiciones de analfabetismo junto a la elevada proporción de población con NBI con respecto a la media provincial marcan las desventajas sociales de la población del oeste pampeano.

Si bien la producción caprina es poco representativa en el contexto provincial, si lo es para la mayoría de la población que habita en los departamentos del oeste de La Pampa. El 92,15\% del total de caprinos en La Pampa se encuentra en el Oeste Provincial y alrededor del 10\% de los bovinos. En el departamento Chalileo prevalece hoy la actividad bovina sobre la caprina y ovina, habiendo sido a principios del siglo pasado uno de los mayores productores de estos últimos (Dirección General de Estadísticas y Censos: 2008 en García L. 2017). El departamento Limay Mahuida redujo drásticamente los rodeos caprinos desde el año 2002, de manera simultánea aumento la cantidad de bovinos. Cuando inicia el PSA la predominancia ganadera en el Oeste era caprina, con elevada población viviendo en los puestos y las condiciones de vida se presentaban más acuciantes que las representadas por los datos censales actuales. EI PSA fue ideado, según cita la resolución de su creación, para intensificar acciones que de promoción y asistencia de quienes se encontraban en situaciones de carencia económica y social (PSA, 2001).

\section{EI PSA en territorio del Oeste pampeano}

\section{El contexto del Programa}

Las intervenciones del Estado en el área de estudio y el contexto amplio del Oeste pampeano comenzaron avanzado el siglo XX. . Sintetizamos algunas de las acciones estatales citadas en García, L. (2016) vinculadas con: la dotación de infraestructura viaria, equipamiento en asentamientos urbanos con mejoramiento del hábitat, colocación de paneles solares en los puestos, construcción de escuelas hogares entre otras cuestiones materiales; todas ellas indisociadas de lo que identificamos como inmaterialidades en el territorio. Esas transformaciones invaden distintas esferas sociales que se entrelazan y se manifiestan en nuevos escenarios de conflicto como el acceso a los recursos, a la propiedad de la tierra, al mercado, a la necesidad de migrar, o moverse rutinariamente, advirtiendo en ello otras relaciones de poder, otras configuraciones de la subjetividad.

La política neoliberal tuvo su máxima expresión en los 90 con: retracción del Estado, ajuste, aumento de la desocupación y mecanismos 
de los organismos internacionales para el diseño y financiamiento de políticas no solamente económicas. En el marco de este escenario global, en América Latina se difunden políticas de desarrollo rural y apoyo a pequeños productores rurales expulsados por el mismo sistema. Mario Lattuada, María Nogueira y Marcos Urcola (2015) desarrollan ampliamente los vínculos entre el concepto de desarrollo, programas de desarrollo rural y sus aplicaciones a través de políticas pública. En el transcurso de las últimas décadas encuentran en ellos una constante idea de identificar lo rural "con una sociedad tradicional y atrasada que debe ser transformada" (Lattuada, M, Nogueira M y Urcola M, 2015, p.29). Esas transformaciones incluyen territorios, grupos sociales, sectores específicos. Esas ideas según los momentos históricos han impregnado al conjunto de los organismos de financiamiento y se traducen a su vez en enunciados de los programas que financian. En la misma fuente se utiliza metafóricamente la mención "parecidos de familia" para representar entre otros ejemplos, los vínculos entre programas financiados por el Banco Mundial -PROINDER-y bases que se encuentran presentes en los programas financiados por los presupuestos nacionales, como el Programa Social Agropecuario o los programas del INTA. Si bien esa constelación "familiar" no implica integralidad alguna, si es interesante rescatar como se impone en las negociaciones BM FAO -SAGyP para el diseño de PROINDER -entre otras cuestiones- la ampliación de la población objetivo que incluye además de pequeños productores, a mujeres, a población aborigen, a asalariados transitorios, promovidos también por otros organismos internacionales de financiamiento de los programas de desarrollo. (Lattuada, M, Nogueira M y Urcola M, 2015, p.31).

EI PSA participó de la formulación del PROINDER, aprobado en 1998 con el nombre de Proyecto de Desarrollo de Pequeños Productores Agropecuarios (ver Figura II). En La Pampa se convierte además, en el canalizador de los fondos de este Proyecto que tenía como destinatarios a pequeños productores minifundistas con necesidades básicas insatisfechas ${ }^{10}$. Las líneas de PROINDER en coincidencia con las acciones desarrolladas por PSA priorizaban el desarrollo de la organización grupal y el fortalecimiento de su autogestión, promoviendo la asistencia técnica donde incluían el financiamiento no rembolsable. Al enfocarse en el sistema productivo campesino este programa enunciaba mecanismos que garantizaran la incorporación de grupos vulnerables, ¿quiénes? "Indígenas, mujeres y jóvenes". A nivel global aparece como "preocupación" la dominación masculina en las discusiones públicas que tienden a no reconocer ni respetar los asuntos específicos ni las opiniones de la mujer. ${ }^{11}$ Advertían entonces sobre la invisibilidad de grupos vulnerables y marginados que no eran tenidos en cuenta dentro de la planificación comunitaria tradicional o en las estruc- 
turas de gestión dada la discriminación y el distanciamiento social al que se ven sometidos. Por lo tanto, sugerían precisar esfuerzos especiales para consultar "con las mujeres y poder así recolectar sus opiniones y requerimientos" (Dahl-Ostergaard: 2003, p. 4). Estos proyectos, aunque actúan de manera puntual requirieron necesariamente de contextos institucionales (Estados) que garantizaran su viabilidad haciéndolo extensivo a través de planes de promoción, sin lo cual estas experiencias se convertirían en esporádicas y discontinuas.

\section{La aplicación del Programa}

El Programa Social Agropecuario (PSA) creado en 1993 como una política sectorial para los "pequeños productores minifundistas" sufrió los avatares de la discontinuidad en las políticas, y los sucesivos cambios de funcionarios en el área agropecuaria. La figura № 2 (Estructura Ministerial) da cuenta de esos cambios vinculados con la trayectoria del Programa.

Figura $\mathbf{N}^{\circ} 2$. Estructura Ministerial

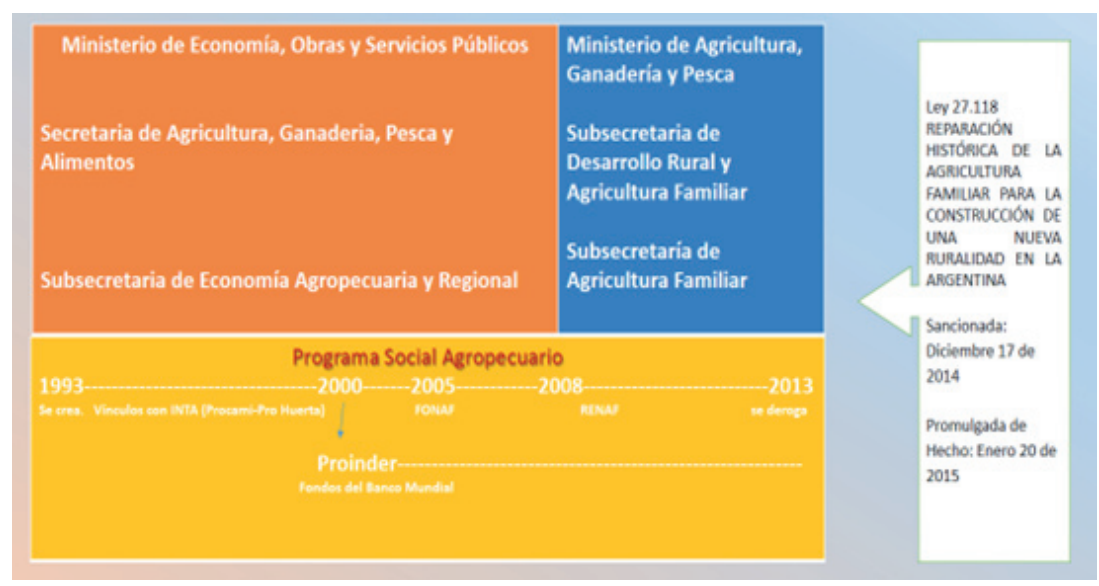

Fuente: elaboración propia.

Su fortaleza estuvo en la perspectiva de trabajo conjunto y el funcionamiento descentralizado con activa participación de los "beneficiarios y beneficiarias, redes de instituciones, organizaciones y organismos interjurisdiccionales los cuales "favorecieron que sus líneas centrales de filosofía y de sus normativas pudieran preservarse" (PSA, 2001, p.7). El financiamiento provino del Estado Nacional, a través de la ley de financiamiento desde el Ministerio de Economía y su par de Salud y Acción Social. A 
través de las Provincias se ejecutaron los Fondos Rotatorios dirigidos a créditos para productores.

A partir del año 2000 se liberan fondos del Banco Mundial para el PROINDER (Proyecto de desarrollo de pequeños productores agropecuarios) los cuales se ejecutan a través del PSA.

La Coordinación del programa en la Provincia de la Pampa estuvo a cargo del Ing. Julio Baggato junto al Equipo técnico de apoyo y a la Unidad Provincial, compuesta por productores del área bajo riego y de secano, INTA y Ministerio de la Producción de La Pampa. El Programa Social Agropecuario (PSA) involucró a más de 100 familias productoras en este sector de la provincia y a diferencia de otras intervenciones gubernamentales, arbitró un seguimiento personalizado de los grupos haciéndolo desde una visión que integró aspectos de la sustentabilidad ambiental, económica y social.

Los objetivos generales del programa se vincularon con: el mejoramiento de las actividades productivas y de los niveles de ingreso, con la generación de espacios de participación que facilitaran la organización de familias productoras, a los efectos de que pudieran asumir su propia representación y desarrollar capacidades de gestión incluso con otras jurisdicciones del Estado. ${ }^{12}$ En el año 1998, el PSA registraba 22 grupos en los departamentos aledaños a los estudiados: Puelén y Chicalcó. Recién en 2006 comienzan las reuniones en El Paso, Santa Isabel, y puestos del Departamento Chalileo en vista a organizar grupos de productores (consorcios y luego asociaciones).

En los boletines de difusión del PSA- INTA (Ecos del Oeste, 1998) se deja constancia de las demandas presentadas por los productores para elevar a la Unidad Provincial: la preocupación por la situación de ocupantes precarios y tenencia de la tierra, la necesidad de mejorar los medios de comunicación (radios y arreglos de caminos) como también la ayuda para arreglos de viviendas afectadas por temporales. Entre otros puntos advertían la necesaria administración por parte de los propios productores del manejo del alimento balanceado y el botiquín veterinario, en cada lugar.

El equipo de la Subsecretaria que llevó adelante el Programa reunió a unas 10 personas que conformaron los equipos técnicos territoriales divididos en seis zonas de la provincia, junto a otras diez referentas a cargo de: Capacitación, Promoción y Seguimiento de créditos y Asistencia Técnica, Comunicación, Seguimiento Plan de participación, RENAF- FONAF y grupos Sujetos de Derechos (luego paso a Grupos Vulnerables, entre ellos: Mujeres).

En la provincia de La Pampa los beneficiarios mayoritariamente fueron varones, aun así, el grupo de especialistas que encausaron el seguimiento del proyecto realizan la convocatoria extensiva a "sus esposas" creando 
el vínculo, en la formación de grupos y capacitación. En ese sentido, el Ingeniero Bagatto que llevó adelante las líneas de acción del PSA hasta el año 2013 rememora algunas situaciones citadas en (García 2017) como antecedentes que definieron la particular estrategia de asociar a los y las productoras: la convocatoria de participación de toda la familia. Esto invito a que en las primeras reuniones las mujeres se incorporaran sin "hablar" hasta que los encuentros que se sucedieron fueron ganando lugares y posibilidades de representación.

En el año 1998, año de inicio y acople del PROINDER al PSA, se comienza a hablar de las mujeres ${ }^{13}$ :

a pedido de un grupo de instituciones preocupadas por la situación de las mujeres en las zonas rurales el 15 de Octubre de 1998 se celebró por primera vez el día Mundial de la Mujer Rural. Por eso el PSA hace llegar un saludo a todas las mujeres que desempeñen el papel fundamental de cuidar la familia, atender los animales, la huerta y todas aquellas actividades que realizan en el predio (PSA-INTA 1998, p.10).

Pasó más de una década del programa en la Provincia para que se reconociera a las mujeres y jóvenes como sujetos de derechos, pero "vulnerables". A partir del año 2008 las mujeres iniciaron su participación en los grupos y, muchas de ellas, lideraron las asociaciones ${ }^{14}$. Dentro del equipo técnico, la referente de este espacio llevó adelante acciones junto al Ministerio de Salud de la Provincia, a solicitud de las mujeres del Oeste que no podían acceder a estudios ginecológicos y médicos en general. De esta manera, junto a la promoción y capacitación de diferentes actividades productivas y artesanales se dieron talleres, asistencia y encuentros de mujeres con temáticas vinculadas a derechos y cuidados de la salud. Las actividades se sustentaron con periodicidad hasta 2011 con iniciativa, contactos y trabajo de tres referentas del equipo y el respaldo del coordinador del programa. Cuando se deroga el Programa en el año 2013 se desafectan a las referentas y otros agentes del territorio. Esto queda refrendado por el funcionario a cargo de la Subsecretaría (año 2013) quien manifestó expresamente que el trabajo con las mujeres "se terminó" (Referente equipo técnico del Programa-año 2016).Pero no solo se termina el trabajo con las mujeres sino con toda la red de acciones en territorio junto a otras instituciones.

Casi todas las crianceras han tomado la posta de las asociaciones... aunque ahora está todo parado... Desde hace cuatro años, ningún organismo oficial las acompañó... sólo un poco el INTA... han quedado todas estas organizaciones incipientes... que necesitan acompañamiento continuo, si no se quedan... Por ahí toman decisiones por dentro pero no participan 
con otras... eso lo hacíamos nosotros... (Responsable de Programa, año 2016).

Las acciones conjuntas con el INTA y el Ministerio de la Producción al año 2016 solo intentaron reestablecer los contactos en el territorio, aunque sin financiamiento y sin programas específicos. ¿Qué quedó de toda esa experiencia? García (2017) interrogó acerca de los términos institucionales de participación en las asociaciones y en algunos casos advirtió un reforzamiento de patrones clientelares. Aun así rescata las acciones colectivas que resultan "novedosas" en este espacio y en la vida cotidiana de las mujeres que suman a su actividad de crianceras cierta forma de "activismo".

\section{La experiencia (asociativa) de las mujeres en el marco del PSA}

Recuperamos escenas en el sentido planteado por Pecheny y Palumbo (2017) como forma que relata lo social, facilita la sistematización y el análisis de los datos. Tomamos de un artículo anterior (García 2017) alguna de esas escenas que redefinieron direcciones, convocatorias, agenda de problemas en el marco del PSA y también reconocimiento de nuevos roles en los espacios domésticos-familiares. En este sentido es central reconocer actores/as implicadas en el "hacer" de este programa. En el artículo citado se describe el caso de las dos asociaciones incluidas en el presente artículo. Las escenas de reuniones convocadas por el programa y dirigidas a productores se dislocan a la presencia de las familias, y ante la negativa de los varones a participar, el "ofrecimiento" a las mujeres para presidir las asociaciones. Las razones mencionadas en la misma fuente refieren a que el manejo por parte de las mujeres, de la escritura rápida, la disposición a viajar y la consideración de que estas actividades aportarían beneficios a la unidad de producción familiar se anclaron como parte de las tareas productivas-reproductivas que en estos territorios son indisociables. Claro que la participación no solo se vinculó a los aspectos técnicos de los rodeos, sino que las demandas se integraron en la complejidad productiva-reproductiva. En los ámbitos de discusión nacional como el FONAF se incluyen temas vinculados con la defensa de la tierra y la libre circulación, cuestiones que ampliaron el horizonte de las demandas además de convertirse en escenarios novedosos de participación. En las voces de las dos presidentas de las asociaciones (El Paso y El Salitral) se reconocen estos espacios de participación como favorecedores de cambios en "la forma de ver las cosas", experiencias que incorporan en sus proyectos familiares y comunitarios. Resulta central dentro de esos cambios la habilitación de la palabra: "poder hablar y por eso no se pierde el respeto" como argumenta Susana Cuello (Asociación El Salitral) esto dio pie a otros cambios, en lo comunicativo, en el abordaje y 
la resolución de problemas entre agentes del programa y productoras y productores. También interesa rescatar cómo estas nuevas escenas de participación de las mujeres impactan en las relaciones familiares. García (2017) destaca que la participación "institucionalizada" es novedosa no solo para las mujeres sino para toda la población del oeste pampeano. Este terreno de acción social contribuyó a crear y sostener formas de cooperación social. El ámbito de participación cambió la dinámica de sus días, los viajes a Santa Rosa, a Buenos Aires; o a los puestos, algunas veces lo hacían solas y otras con sus esposos sobre todo cuando convocaban a asambleas. A ello se suma la propia enunciación de las mujeres en resaltar relaciones de cierta paridad en la familia a la hora de involucrarse en actividades que defienden los bienes comunes. Este particular contexto las coloca en un doble juego que las posiciona en el lugar público, pero a la misma vez anclado en roles tradicionales de sostenimiento y resguardo de la reproducción doméstica. Aunque es incipiente el reconocimiento público, el mismo se funda en esas relaciones de cierto equilibrio que experimentan en las unidades domésticas y que otorgan cierto empoderamiento a las mujeres presidentas de estas asociaciones. Este nuevo campo reconfigura estrategias particulares en la consecución del programa ya que deben pensar en la integralidad demandada por las mujeres presidentas de las asociaciones.

\section{El "decir" y "fabricar" del Programa Social Agropecuario: imágenes de unos y necesidades de "otros"}

Mencionamos con anterioridad las condiciones ambientales del lugar que forma parte de la diagonal árida del Oeste de Argentina con precipitaciones anuales inferiores a 400 milímetros, sin presencia de escurrimientos fluviales permanentes y con calidad de agua no siempre aptas para consumo humano y animal. Muchos puestos requieren asistencia por parte de los municipios cercanos del agua para consumo. Este punto es el primer acto de interpelación por parte de las y los productores. Cuando los técnicos y agentes del programa Social Agropecuario llegan a Paso de los Algarrobos con la intención de formular un proyecto en colaboración con la Asociación El Paso, las ideas giraban en torno a la concreción de corrales e infraestructura para realizar destetes anticipados de terneros. Fue la presidenta de esa asociación Marta Álvarez (M.A) quien plantea las necesidades desde el lugar: priorizar el acceso al agua, disponer de un transporte propio para autogestionar su distribución y fundamentalmente mejorar las rutas y accesos a los puestos entendiendo que sin ellos se imposibilita la tarea. La falta de pasto, frutos y hojas de la flora local asociadas a temporadas de déficit de agua (pluvial-de escorrentía) acompañadas de temperaturas extremadamente elevadas o bajas fueron replanteando el 
proyecto de hotelería de terneros por asistencia con alimento balanceado en las unidades de producción.

En la Asociación El Salitral se suma otra demanda vinculada con el manejo de los rebaños: la amenaza de predadores que a la vez están protegidos por el área de Fauna Silvestre de la Dirección de Recursos Naturales (LP). Es su presidenta (S.C) la que incita a que el programa arbitre reuniones con la esfera provincial para poder acordar acciones. Los diálogos con las reparticiones del gobierno provincial distan de ser compatibles con las orientaciones que toma el PSA. Es que la experiencia de este programa tuvo un componente central y es, quién llevó adelante este programa. En ambas presidentas existe reconocimiento a las prácticas de diálogo que sostiene el responsable del PSA y eso les permitió visibilizar y canalizar sus demandas. En otros programas, la idea de intervenir en el territorio desde lo productivo ha sido muy rígida y chocó con las realidades y prácticas del lugar.

(...) Bagatto sabe los que nos pasa, sabe mucho y tiene otra visión...en cambio cuando vienen los chicos del Ministerio, te quieren cambiar las formas: por ejemplo el chivo por el cordero... Nosotros nos criamos así, y yo les paso la forma a mis hijos y ellos lo harán con los suyos con la cultura, las enseñanzas (Productora).

En García (2017) se menciona que la intención original del programa, de asistir en el mejoramiento productivo y en las condiciones de vida se creó a partir de preconceptos de homogeneidad en cuanto a características de deprivación material y también productiva, que debieron redefinir en el proceso de interacción con los grupos del lugar. El mismo proceso de escuchar las necesidades de la gente también redefinió la metodología de acción del programa en el territorio. Los antecedentes de otras intervenciones de programas provinciales y nacionales vinculados con mejoramiento genético y manejo sanitario de rodeos fueron dirigidos puntualmente a los productores. Los resultados del bajo impacto de las innovaciones no tuvieron en cuenta que quienes gestionan la actividad ganadera menor en el Oeste son las mujeres. La reconfiguración de esas imágenes acerca de quiénes podían gestionar y mediar en los grupos fue un buen aporte para la experiencia de las asociaciones diez años después

Surgen de las mujeres porque en general, aunque parezca esté atrás del hombre, es la que toma las decisiones! Nosotros queríamos involucrar a todos, y ellas se destacaban y los hombres lo reconocieron. Tienen mucha polenta y los hombres no tanta, entonces le "pasaron el fardo", como dicen ellos. (Responsable de Programa. Año 2016). 
Este nuevo escenario ejercitado por el programa identifica aquellas prácticas que no incluye las condiciones de las y los sujetos. Son las mujeres las que registran las situaciones de injusticia intersectadas por varias dimensiones sociales y territoriales.

pero tenemos un técnico que lamentablemente acá no nos sirve...es chocante, con los que no saben leer y escribir y acá la gente es muy humilde... por ahí le dice, vos no podes ir, sabes leer? Son los técnicos representante de agricultura familiar de los productores, ahora le vamos a elevar una nota a Julio porque no nos anda acá, hemos querido presentar proyectos y hemos tenidos que rogarle que venga, que nos apoye, nosotros necesitamos una persona que nos respalde... (S.C El Salitral Año 2012).

Junto a O'Donnell (2004) reconocemos que salvo excepciones, el Estado latinoamericano se ha presentado desde siempre selectivo, con trato discrecional, desoyendo a buena parte de la población.

Ha sido habitual (y aún con regímenes democráticos en no pocos casos lo sigue siendo) la doble discriminación implicada por la negación a mucho de sus derechos junto con el otorgamiento de privilegios y la exención de obligaciones a otros; el trato descomedido, cuando no violento por parte de diversos funcionarios estatales; y las dificultades no pocas veces interpuestas al acceso a servicios estatales fundamentales, educación, salud y justicia incluidos. Esta cara del Estado niega de hecho una ciudadanía, que propiamente entendida, se pone en juego no solo votando sino también en estos encuentros y desencuentros con sus burocracias y su sistema legal (O'Donnell 2004, p. 179).

En el caso de este programa, tanto "el sujeto" de intervención como la metodología de interacción entre estos y los fines del programa tuvieron un proceso de rectificación a partir del descentramiento del lugar tradicional que han tenido las intervenciones del Estado en este territorio. Los cambios pensados desde el lugar y sus realidades redefinieron las prioridades junto a las productoras/es quienes además reconocieron valores etnocentrados. De esta manera el campo de las Asociaciones muestra el conflicto de interés particular del programa con el interés particular y general de los y las productoras pero también conflictos de "sentidos" hacia "el otro". Esto potencia la idea de pensar al Estado más allá del conjunto de burocracias, de su sistema legal y de foco identitario colectivo. Para Echavarri (2018) es pensarlo a partir de las prácticas, como campo de disputa entre sus presupuestos -que sustentan adhesión a los derechos humanos- y las prácticas concretas; entre el "decir y fabricar" y otros modos de organización y concepciones de mundos. Para esta autora la administración desde el Estado no puede separarse del sentido político, 
los procesos administrativos son procesos políticos y desde el lugar de los administradores, en este caso extensionistas, referentes entre otros, no están libres de valores y actores políticos. El enfoque de derechos en las políticas públicas politiza la administración de derechos de quienes no siempre estuvieron incluidos. Aquí es clave entender las políticas públicas como procesos dinámicos que interactúan entre actores sociales y estatales, con intereses particulares y portadores de recursos clave alrededor de cuestiones particulares (Ase, 2019).

La experiencia del Programa Social Agropecuario impactó en quienes pensaron acciones y actuaron específicamente en territorio junto a las familias productoras. La inclusión de las mujeres como sujetas de derecho en el Programa fue tardía y en ocasiones la lectura de acciones particulares y la convocatoria hacia ellas no respetó la integralidad de la perspectiva de género. En el caso de la Provincia de La Pampa fueron las mujeres crianceras - pequeñas productoras que enunciaron sus demandas particulares y garantizaron con su participación las formas organizativas locales y representación en foros y encuentros extra locales. Es ese protagonismo el que invita a gestores del Programa a habilitar una línea de acción para estos "grupos vulnerables" y rescatar el "decir" del mismo alrededor de las "mujeres campesinas". Entendemos que estas relaciones de fuerza que se disputan al interior estatal, han construido demandas a partir de que quienes en territorio enuncian sus principales problemas y necesidades. En este sentido Ase (2019) advierte que la renovación de la gestión pública también se ha vinculado con la cultura de derechos desde la cual los agentes de la Administración Pública son titulares de deberes, donde se sustituye el control jerárquico por la coordinación democrática, donde la ciudadanía debe recibir beneficios iguales, generando acciones positivas hacia grupos desfavorecidos y una administración fundada en el empoderamiento del sujeto.

No es objeto de este trabajo identificar toda la acción pública en el territorio del Oeste pampeano pero la entrada al mismo da cuenta de ausencias continuas, presencias recortadas, fragmentadas y discontinuas por parte del Estado en sus distintas jurisdicciones. Los ensayos acerca de las políticas públicas pensadas desde los derechos humanos deberían según Ase (2019): tener continuidad, contener a las personas y los derechos en el centro del diseño de las políticas públicas. ${ }^{15}$

El Oeste pampeano es muestra de los disloques en las cuestiones prácticas de las políticas públicas. Desde 2013 se acentúan en el territorio los problemas vinculados con cuestiones productivas, el "desarmado" de grupos y con ello la inexistencia de capacitaciones, y asistencia en los lugares. Las demandas individuales de las mujeres corrieron, por un lado, por canales ejercitados en tiempos de las asociaciones aunque no siempre 
lograron resolución o acompañamiento y en otros casos por sus propios medios y recursos.

\section{La cooperativa La Comunitaria en el Oeste}

En el año 2015 llega a Santa Isabel, un grupo cultural reunido en una Cooperativa para relevar voces sobre la problemática del Atuel y de ello surge la obra "Atuel Sed que crece". Esta intervención comenzó a agrupar a puesteras/os -crianceras/os del lugar que luego conformarían una cooperativa. A fines de 2018 conforman el grupo con "70 colaboradoras/ es", bajo la organización de la Cooperativa la Comunitaria ${ }^{16}$ con sede en General Pico, La Pampa. Uno de los objetivos de esta cooperativa es fomentar el espíritu de ayuda mutua entre los asociados y cumplir con el fin de crear una conciencia cooperativa (Art. 3) más allá del rescate y promoción cultural. Con clases de murga, acordeón, guitarra, un merendero y un pequeño emprendimiento de cría de aves en un terreno prestado ${ }^{17}$ a las afueras de Santa Isabel, se inician las primeras acciones. También llevan sus productos (quesos, dulce de leche y artesanías entre otros) a una reunión ampliada de organizaciones en la Ciudad de General Pico. Quienes se hacen cargo de la organización local son dos pequeños productores y una pequeña productora.

La llegada al Oeste del grupo que conforma la Cooperativa viene del contacto con un docente, productor y militante del lugar que plantea la preocupación por el abandono en términos de atención de problemas locales y la situación de familias que evaluaban dejar el campo. A su vez se encuentran con un escenario particular: son las mujeres de distintas generaciones que resisten en los puestos. Los problemas también se registran en el pueblo. Las familias crianceras que han migrado a Santa Isabel enfrentan el desempleo y la imposibilidad de poder generar sus propios alimentos en el pueblo, de ahí que surge la iniciativa de atención colectiva de la producción de pollos y huevos junto al comedor. Quienes siguen en los puestos presentan los históricos problemas de falta de agua y pasto. Ese mismo año solicitaron a los municipios de Algarrobo del Águila, Santa Isabel y Limay Mahuida el transporte para poder traer maíz ante la falta de alimento para los rodeos de chivas. La ayuda no llegó por esta vía sino que llovió y pudieron afrontar la emergencia.

Nos preguntamos iqué es lo que aglutina-amalgama esta nueva experiencia asociativa? Esta Cooperativa de trabajo agropecuaria es parte de la rama rural del Movimiento de Trabajadores Excluidos (MTE) que nuclea organizaciones de campesinos/as, pequeños/as productores/as y comunidades originarias del país en vista a mejorar la calidad de vida y de trabajo de quienes producen los alimentos. EI MTE promociona y fortalece redes de comercio justo y alternativas solidarias junto a la "construcción de una 
gremialidad (...) en torno a los excluidos y excluidas del campo en Argentina, donde los/as pequeños/as productores/as y los/as campesinos/as sean protagonistas de las propias luchas y los propios reclamos, en solidaridad con otros sectores excluidos de la sociedad" (MTE). Esa gremialidad genuina está vinculada con un espacio más amplio que es la Confederación de Trabajadores de la Economía Popular (CTEP) ${ }^{18}$.

La Economía Popular (EP) surge cuando los sectores excluidos del mercado laboral crean su propio trabajo. En algunos casos también crean sus propios circuitos de comercialización, como ferias o venta ambulante. Las unidades de la EP involucran un amplio campo de trabajadores/as independientes que van desde empresas recuperadas, grupos comunitarios de base, medios de comunicación comunitarios, núcleos de agricultura familiar, comunidades de pueblos originarios entre muchas otras organizaciones sin fines de lucro. En 2015 finalizando el mandato de Cristina Fernández se aprueba la Personería Jurídica de la CTEP, con la Resolución 1727 del Ministerio de Trabajo, reconociendo desde el Estado la existencia de trabajadores de la EP y su derecho a la organización. En el año 2016 ante el contexto de inmediato ajuste del gobierno de Mauricio Macri se da la Marcha de Emergencia Social y Laboral luego de la cual se firma el acuerdo de la Emergencia Social. Alguno de los puntos fueron la actualización de los montos que percibían los Programas Sociales y Asignaciones, aumento de las partidas para comedores y merenderos. Ese año se aprueba por consenso la Ley de Emergencia Social, que "implica la creación de una nueva institucionalidad acorde con las necesidades de la Economía popular: 1) Creación de un Registro Nacional de la E. P. (RENATREP) 2) creación del Consejo de la EP 3) Financiamiento y 4) La creación de un Salario Social Complementario (SSC) y la conversión de los Programas Sociales al SSC. Esto último es un concepto estratégico porque modifica el status de los y las beneficiarias pasivas a trabadores y trabajadoras activas. (CTEP) $)^{19}$. "La novedad es que después de 30 años el Estado acepta que no se trata de "efectores sociales", "microemprendedores", "beneficiarios", "titulares de derecho", "subocupados" o "desocupados", sino de trabajadores (Grabois, 2016, p. 17).

Abramovich (2006) entiende que el enfoque de Derechos Humanos apunta esencialmente a otorgar ese poder por la vía del reconocimiento de derechos. "Una vez introducido este concepto en el contexto de la adopción de políticas, el punto de partida para formular una política ya no es la existencia de ciertos sectores sociales que tienen necesidades insatisfechas, sino fundamentalmente la existencia de personas que tienen derechos que pueden exigir o demandar, esto es, atribuciones que dan origen a obligaciones jurídicas para otros y, por consiguiente, al establecimiento de mecanismos de tutela, garantía o responsabilidad" (Abramo- 
vich, 2006:40). Quienes integran la Cooperativa hoy son 120 crianceras/ os, pequeñas/os productores familiares con residencia en los puestos, en el pueblo y en algunos casos con doble residencia. Solo quienes sean mayores de 18 años y no tengan asignación, pensión o jubilación pueden acceder a la "tarjeta de la comunitaria". Llegaron a estar cubiertos 95 socios y socias, de los cuales hoy perciben el SCC 11 productoras y 44 productores; el resto obtuvo jubilación o "trabajo en blanco" según datos de su presidenta Alejandra Domínguez (A.D). En la actualidad el salario social complementario es de 10.500 \$ que en muchos de los casos es el único ingreso monetario y lo usan como menciona una integrante de la cooperativa "para comer y cargar combustible para ir al pueblo a llevar los chicos a la escuela o buscarlos". Es indudable que "La Comunitaria por ser parte de un movimiento social, llegó con algo concreto que fue el SSC... que para algunas familias significa: quedarse en el campo" estas son las palabras de Carlos Alainez (C.A) militante del MTE y compartida por muchas de las productoras entrevistadas.

\section{Los movimientos sociales en la gestión de la SAFCI}

A partir del año 2020 se redefinen representaciones en la organización de la Secretaría de Agricultura Familiar, Campesina e Indígena (SAFCI) del Ministerio de Agricultura, Ganadería y Pesca. La delegación La Pampa de la Subsecretaria de Agricultura Familiar y desarrollo territorial se organiza como en todo el país con la inclusión de movimientos sociales de base territorial. Así el MTE junto a otros movimientos como Movimiento Evita y la Corriente Clasista y Combativa coordinan la Zona oeste, centro y norte respectivamente.

Las coordinaciones se efectivizaron a principios del corriente año, es por esto que solo mencionamos las convocatorias de algunos programas. Nos interesa destacar que quien promovió los procesos asociativos a través de La Comunitaria hoy está al frente de la Coordinación Oeste: Carlos Alaines. Su interés está puesto en recuperar "la época de gloria que supo tener esta repartición entre 2008-2014... recuperar nivel de trabajo, presupuesto y recursos" reconocidos por la población del Oeste. La habilitación de distintos programas de promoción impactó en nuevas decisiones para la cooperativa. Esto definió ( con muchas discusiones) la necesidad de constituir una Cooperativa de Trabajo Agropecuaria Regional Limitada dentro de La Comunitaria para poder presentar proyectos al Programa de Promoción del Trabajo, Arraigo y Abastecimiento Local (PROTAAL) y (PISEAR) Proyecto de Inclusión Socio-Económica en Áreas Rurales. Alejandra Domínguez recupera de esas discusiones la necesidad de acceder a la compra de forraje accesible sin depender del precio del mercado y la 
gestión autónoma de su distribución. Para ello considera imprescindible disponer de un transporte que pueda además completar el circuito de comercialización. En ese camino se orientan las presentaciones de la nueva cooperativa a las distintas propuestas de la Secretaría. Los objetivos del plan estratégico 2020-2024 de la SAFCI están enmarcados en una "planificación con perspectiva de género incluyendo a las juventudes rurales, buscando el cuidado del ambiente y la promoción de la agroecología". En este sentido surgió el programa "En Nuestras Manos" con tres líneas: Tecnología para las mujeres rurales, Asistencia pos pandemia y Refugios de Mujeres víctimas de violencia. La convocatoria nacional se abrió en el año 2020 sin tener a las y los responsables provinciales designado, esto no jugó a favor para que pudieran presentarse a tiempo. Hoy se despliega un escenario diferente ${ }^{20}$, aunque, habiendo programas con perspectiva de género y condiciones para presentarse en las convocatorias, persisten mecanismos burocráticos que impiden poder acceder a los mismos.

\section{Sostenido activismo de las mujeres en la cooperativa}

La comisión de la cooperativa de trabajo agropecuaria de Santa Isabel no ha tenido renovación este año producto del contexto de pandemia. De los siete cargos dos son ocupados por mujeres: el cargo de presidenta y tesorera. Dentro de la cooperativa hay quienes vienen transitando la experiencia asociativa y otras que lo hacen por primera vez como es el caso de las mujeres de la comisión. La idea de pensarse como trabajadoras/es de la economía popular es una de las cuestiones que también se pone en tensión al interior de la dinámica de la cooperativa. Se advierte el fuerte liderazgo de las mujeres y jóvenes en las voces que formulan los problemas, sea en ámbitos de difusión o de discusión asamblearia. ${ }^{21}$ Los problemas que enuncian a nivel productivo son los mismos que se planteaban en los años de vigencia de las asociaciones: retención de los rodeos, sanidad, valor agregado de su producción y la comercialización. Las asambleas mensuales que coinciden en algunos casos con el cobro de la tarjeta reúnen a las familias, y han generado una reciente adscripción identitaria alrededor de la "comunitaria".

En la comunitaria aprendes un montón de cosas. La gente de campo por acá está acostumbrada a trabajar por sí sola, pelea por lo suyo....pero somos muchas/os que estamos en problemas y con el mismo propósito que es "no abandonar el campo". Aprendes a trabajar por todos y es diferente... porque pensas "ya no me van a joder más", por ejemplo cuando compramos el alimento o vendemos los chivos y que paguen lo que debe ser. (A.D Productora de la Cooperativa). 
Esta fortaleza en los lazos comunitarios a veces es minada por problemas estructurales. En el área estudiada se presenta un panorama que agrava la situación: las personas envejecieron, las mujeres sobreviven a los varones y quedan en los puestos, las enfermedades no se atienden debidamente y no disponen de recursos monetarios entre muchos otros problemas. Seguimos encontrando en los relevamientos de información en campo a mujeres sin acceso a jubilaciones o pensiones por enfermedades y sin acceso a la educación. La imposibilidad de acceder a esos derechos va desde la desinformación a la imposibilidad de afrontar viajes a los centros de salud o al propio ANSES en Santa Rosa, por los costos de movilidad. Esta cara del Estado según O'Donnell (2004) niega de hecho una ciudadanía, aquí se evidencia el desencuentro entre su burocracia y su sistema legal. La cuestión seria avanzar hacia niveles, para llamarlo de alguna manera, decentes de ciudadanía civil y social. Estos problemas, aunque por su puesto las incluyen, no se refieren solo a diversas minorías, los sufren también verdaderas mayorías, como las que constituyen en muchos de nuestro país los pobres, y por cierto las mujeres. Es una deuda en la economía social y en el resto de las políticas centrar la atención en la reproducción y el cuidado de la población generalizadamente en manos de las mujeres y posicionarla como responsabilidad social.

Para todos estos sectores se trata de las inexistencias de diversos derechos, de la falta de implementación de otros, y de múltiples prácticas, algunas sutiles y otras violentas de discriminación. "Esto vuelve al tema de la agenda, del juego democrático y por cierto de la capacidad de compromiso y movilización con que las fuerzas democratizantes impulsen avances que, podemos estar seguros, no ocurrirá espontáneamente" (O'Donnell, 2004, p. 180). En este sentido el mismo autor insiste, en que resolver esto como acto de justicia, también "implicaría "empoderar" (empower) a los sectores populares para que estén en mejores condiciones de ir definiendo y redefiniendo sus propios intereses e identidades esto sería a su vez expresión de la ampliación de la agenda pública, de la vitalidad del juego democrático y de la receptividad de un estado que se iría ensanchando en ese proceso" (O'Donnell, 2004, p. 180). Lejos de esta tesis sustentada por este autor, en estos territorios del oeste pampeano se desarrollan acciones de lo público en dos esferas que no se tocan y donde las y los sujetos son quienes se encuentran entre ellas: nos referimos a la Cooperativa inserta en la economía popular y a las políticas del ámbito provincial. Se da por un lado el cambio paradigmático de reconocer la economía popular, a las y los trabajadores en ella y la inclusión a través del financiamiento. Por otro lado, acciones desde las políticas de la provincia que siguen sosteniendo a la población como vulnerable, con políticas fragmentadas, discontinuas y que no llegan a toda la población. Entre estas intervenciones una pobla- 
ción en condiciones de desigualdad extrema: ambiental, productiva, social con respecto al Este de la Provincia. Entonces el desafío en coincidencia con Echavarrí (2018) es pensar el enfoque de derechos en la gestión y considerar "otra manera de construir pensamiento". De aquí la necesidad de observar estas políticas con perspectivas de género(s), de derechos, de pensar nuevas posibilidades de construir problema e incorporarlo en la agenda pública, de mirar nuevos sujetas políticas emergentes, de ligar reconocimiento y redistribución, como también habilitar otras construcciones desde el derecho y desde el Estado.

\section{A modo de cierre}

Los territorios del oeste Pampeano tienen marcas de acciones y omisiones por parte del Estado. El análisis de las formas participativas de las mujeres y familias en este recorte territorial del oeste pampeano tienen relación directa con intervenciones estatales. Los territorios a escala global reflejan las contradicciones del sistema capitalista: en simultáneo a la hegemonía del capital camina el paradigma de los Derechos Humanos y en este contexto se esbozan estas políticas para "pobres, vulnerables". La estructura fundante del PSA ligada luego a PROINDER ensaya un nuevo paradigma en las políticas de desarrollo rural vinculadas con la participación y la visibilidad de las mujeres. En la práctica evidenciamos la difícil convivencia entre las estructuras fundantes de normalización del Estado y estas dinámicas emergentes de la perspectiva de Derechos. El lugar de las mujeres fue ganado por las propias crianceras y pequeñas productoras de la agricultura familiar. La revisión de las propias estructuras de quienes llevaron adelante el programa redefinieron prioridades y agendas planteadas por las familias de productoras. Con todas las interrupciones presupuestarias y de cambios del organigrama ministerial donde se radicó el PSA, fueron los vínculos sustentados desde este lugar con: INTA, Municipios y dependencias del Gobierno de la provincia de La Pampa (Salud, Ministerio de la Producción) los que ensayaron cierta integralidad, transversalidad, interseccionalidad e interjurisdiccionalidad. Actualmente se le disputa al Estado todas estas cualidades ausentes en el territorio. El neoliberalismo tiene formas particulares de "fabricar" no es mero contexto como plantea Echavarri (2018). Por un lado, enuncia ampliación de derechos y al mismo tiempo desmantela las estructuras que viabilizaron acciones, desarticula redes y abandona a la población en territorios considerados "no productivos". La gran conquista ganada por el reconocimiento de la Economía Popular y el cambio de paradigma acerca de trabajadoras/es independientes insertos en la E.P abre un campo novedoso como lo fue en su momento el PSA. Las mujeres demuestran a través de su liderazgo y participación 
su lugar en el espacio cooperativo, aunque no hay enunciación expresa de demandas particulares para sus problemas particulares: sobrecarga en las tareas de cuidados en general (reproducción-producción), atención de su propia salud, mejoramiento de las viviendas, entre otras. Ligar reconocimiento y redistribución desde una perspectiva de género(s), integralidad y respeto de los lugares y su gente es la apuesta para pensar intervenciones del Estado.

\section{Referencias bibliográficas}

Abramovich (2006) Una aproximación al enfoque de Derechos en las estrategias y políticas de desarrollo. Revista de la CEPAL, $\mathrm{N}^{\circ}$ 88. Págs.35-50.

Ase, I. (2019). Administración Pública. Enfoque de Derechos Curso de Posgrado: Transformaciones Estatales, Políticas Públicas Y Nuevxs Sujetos De Derechos (ADIUC). Córdoba.

Burijovich, J. (2019). Administración Pública. Enfoque de Derechos. Curso de Posgrado: Transformaciones Estatales, Políticas Públicas Y Nuevxs Sujetos De Derechos (ADIUC). Córdoba.

Comerci, M. E. (2014). Complejidades y diferenciaciones en el territorio pampeano. En, Lluch, A. y Salomón Tarquini C. Historia de La Pampa I. Sociedad, política, economía. Santa Rosa: EdUNLPam. Segunda edición.

Covas, M. R. (1998). Los espacios socioeconómicos de la provincia de La Pampa. HueIlas Revista del Instituto de Geografía de la Facultad de Ciencias Humanas de la UNLPam, № 3. Disponible en: https://repo.unlpam.edu.ar/handle/unlpam/2574

Comerci, E. (2017) Perspectivas en torno al estudio de las estrategias p. 24-48. En Comerci, E. (Compiladora) Estrategias en espacios de borde. Colección Libros Académicos de Interés Regional. EdUNLPam.

de Arce, A.(2020) Desigualdades Instituidas. Género Y Ruralidades En La Argentina (S.XX-XXI) Estudios Rurales No20, CEARUNQ. -En línea-
Ecos del Oeste. Boletín №2 Diciembre 1995. PSA. INTA. PROCAMI INTA AnguilEscuela Hogar No 157.

Echavarri, L. (2018) Estado y Política Pública en clave feminista: Sobre la perpetua pretensión estatal de categorizar el "ser mujer". Crítica y Resistencias. Revista de conflictos sociales Latinoamericanos. Nº8- págs. 1530 Colectivo de Investigación El Llano en Llamas. Córdoba. Argentina-en línea.

García, L. (2015) Aproximaciones al abordaje de la vulnerabilidad social con perspectiva de género en espacios de borde. Departamento Chalileo y Limay Mahuida (Provincia de La Pampa). Revista Huellas No 19 EdUNLPam.

García, L. (2016) Vulnerabilidad social y políticas públicas aplicadas en el Oeste Pampeano. Miradas desde una geografía feminista. Argentina. Villa María. 2016. Congreso. $2^{\circ}$ Congreso AAS y $1^{a}$ Jornadas de Sociología UNVM. Universidad Nacional de Villa María ISBN 978-987-1697-91-5

García, L. (2017) Asociaciones en manos de mujeres p79-118. En Comerci, (Compiladora), Estrategias en espacios de borde, Colección Libros Académicos de Interés Regional. EdUNLPam.

GEOFEMSUR (2020) Memorias del I Encuentro De La Red De Geografías Feministas Del Sur (Geofemsur) Bogotá, D.C., Colombia. Disponible en https:// es-la.facebook.com/198013633543496/ videos/642633583315740/

Grabois, J. (2013) Capitalismo de exclusión, periferias sociales y movimientos populares. Pontifical Academy of Sciences, Scripta Varia 123, Vatican City. 
Grabois, J. (2016) Personería Social. Perspectivas en torno al nuevo régimen de agremiación para los trabajadores de la economía popular - 1a ed. - Buenos Aires: Universidad de Derecho: 16-17 Disponible en http://www.ctepargentina.org/wp-content/ uploads/2017/09/personeria_social.pdf

Lattuada, M ; Nogueira M.E. ; Urcola M (2015) Tres décadas de desarrollo rural en la Argentina: continuidades y rupturas de intervenciones públicas en contextos cambiantes 1984-2014. Ciudad Autónoma de Buenos Aires: Teseo; Universidad Abierta Interamericana.

La Comunitaria -en línea-MTE http://mteargentina.org.ar/

O`Donnell, G. (2004) Acerca del Estado en América latina Contemporánea. Diez tesis para su discusión. Obtenido de http://www. unsa.edu.ar/histocat/instituciones/odonnell.pdf

Ostergaard, T D y otros (2003). Desarrol/o rural de autogestión comunitaria. BID Serie de informes técnicos del Departamento Desarrollo Sostenible. Washington, DC.

Pecheny M. Palumbo, M (Compiladores, 2017). Esperar y hacer esperar, Buenos Aires. Disponible en:https://www.teseopress. com/esperaryhaceresperar.

SAGPyA (2001) El programa Social Agropecuario. Ministerio de Economía.

\section{Notas}

1 Profesora en Geografía. Especialista en Estudios Sociales y Culturales. Profesora Asociada en Geografía de América Latina, UNLPam, Departamento e Instituto de Geografía.

2 Este trabajo se enmarca en el Proyecto "Tramas sociales, estrategias y políticas públicas en los márgenes pampeanos (2000-2020)" dirigido por Maria Eugenia Comerci, FCH, UNLPam.

3 Las geografías feministas proponen "investigar y sacar a la luz la relación que hay entre las divisiones de género y las divisiones espaciales, para descubrir cómo se constituyen mutuamente, y mostrar los problemas ocultos tras su aparente naturalidad" ( McDowell 2000 p. 27).

4 Aclaramos que si bien los términos no son equivalentes los programas refieren a pequeños/as productoras; en el territorio es utilizado y auto referenciado el término Criancero/a; puestero/a y en producciones académicas el concepto Campesino/a refiriendo a los mismos grupos. En todos los casos se enmarcan en sistemas campesinos cuyas fuentes de ingreso son monetarias y no monetarias, problemas con la tenencia de la tierra, venta de producciones para el mercado, producción para autoconsumo, ingresos extraprediales, seguridad social, trabajo familiar entre otros (SAGPyA).

5 Se crea por Resolución N ${ }^{\circ} 158$ del 7 de abril de 1993 y se deroga por Resolución 1164/2013en Noviembre de 2013.

6 EI PSA represento una estructura institucional dependiente de la SAGPyA, no es objetivo en este trabajo el análisis institucional sino su programa de políticas de promoción e igualdad de oportunidades y de vínculos interinstitucionales que identificamos como política pública.

7 Esta Cooperativa conforma la rama rural del Movimiento de trabajadores excluidos (MTE) de la Confederación de los trabajadores de la Economía Popular (CTEP).

8 Relevados en el marco de distintos proyectos del Instituto de Geografía de la Facultad de Ciencias Humanas.

9 La descolonialidad en este caso, debe implicar una práctica que vaya más allá de la teoría, elemento que diferenciaría a nuestras geografías feministas latinoamericanas, en donde la descolonialidad se está construyendo a través de ontologías y métodos aplicados que ubican la práctica política. Pensar en cómo descolonizar el ejercicio académico y la producción de conocimiento, 
debe pasar entonces por la proyección de una pedagogía distinta, que pluralice y subvierta ese conocimiento científico que sigue siendo eurocéntrico y colonial, dando apertura a la diversidad y las disidencias. En palabras de Eloísa Berman (2020), esa pluralización de las epistemologías contiene una pedagogía de la escucha, que abogue por permitir el impacto de la otra y así remover y hacer tambalear nuestros propios marcos y lógicas en la producción de conocimiento $y$ en el accionar político (Memorias GEOFEMSUR).

10 Para conceptualizaciones de pequeños productores ver (Edith Obschatko; María del Pilar Foti y Marcela Román (2007,) Sakoumagkos, Soverna y Craviotti (2000).

11 La plataforma de Acción de Beijing surgida a partir de la Cuarta Conferencia Mundial sobre la Mujer (ONU) en 1995 esboza los ámbitos críticos que constituyen obstáculos para la igualdad de género.

12 Desde sus inicios hasta mediados del año 2000 las acciones en territorio junto a INTA, se vincularon con capacitaciones a quienes producían y promotores de PSA (servicio del rodeo, alimento balanceado, huertas, procesamiento de alimentos, sanidad en el rodeo e instalaciones); implementación de botiquines veterinarios comunitarios; entrega de créditos para compra de cabras y reproductores, mejoramiento de instalaciones rurales (bretes, bomba rosario, invernáculos tubulares, pantallas solares) y para producción de textiles: ruecas y telares. Entre otros instrumentos para llevar adelante los objetivos promovieron líneas de comercialización de productos (bolsa de trueque) y encuentros de productoras y productores a nivel provincial y nacional.

13 de Arce (2020) plantea que en los años noventa, la renovación del enfoque hacia los problemas de Género y Desarrollo busca mejorar la autoestima femenina y trabajar sobre la conciencia de ciudadanía y derechos. Intenta revertir la situación de subordinación de las mujeres y ampliar las oportunidades de acceso al control de recursos y toma de decisiones. (de Arce 2020, p 18).

14 Lattuada, Nogueira y Urcola (2015) plantean que en el año 2008, la creación de la Subsecretaría de Desarrollo y Agricultura Familiar responde tanto a la demanda de las organizaciones de productores nucleados en el Foro Nacional de Agricultura Familiar (FoNAF) como a los postulados del Desarrollo Territorial Rural presente en las propuestas de los organismos internacionales. Es recién en este contexto, que el desafío de incluir la perspectiva de género en las políticas públicas atraviesa todo el proceso de la gestión gubernamental. (Lattuada M, Nogueira M y Urcola M 2015 p.15-16).

15 Junto a ello, potenciar el empoderamiento las personas y de las organizaciones, y el reconocimiento explícito de los marcos normativos de los DDHH (el derecho internacional de los $\mathrm{DDHH}$; realización progresiva y principio de no regresión, igualdad y no discriminación).

16 La Cooperativa La Comunitaria es una cooperativa social y cultural de provisión de servicios. Tiene matrícula nacional otorgada por el INAES desde el año 2012.A raíz de todos los proyectos artísticos, culturales y sociales que se "desprendían" de los grupos de teatro comunitario en cada una de las localidades (Gonzales, Moreno, Rivadavia, América- Pcia. de Buenos Aires,) centralizaron todas las actividades en una figura legal, que posibilitó hacer visible la multiplicidad de trabajos realizados y una apertura muy importante a la comunidad, ya no sólo desde el teatro. Es así como surge la conformación de la "Cooperativa La Comunitaria de Provisión de Servicios Culturales y Sociales Ltda. http://www.lacomunitaria.com.ar/ index.php

17 La "huerta" y tuvo varias localizaciones, inicialmente en un terreno de una asociada y ex presidenta de la Asociación EI Salitral y hoy en un terreno cedido por el Municipio de Santa Isabel. 
18 Es necesario contextualizar para el 2011 un campo de emergencia en el contexto nacional y sobre todo Urbano. Ese año nace la Confederación de Trabajadores de la Economía Popular (CTEP) como una organización gremial independiente de todos los partidos políticos, representativa de los trabajadores de la economía popular y sus familias. El crecimiento a nivel regional latinoamericano y el mejoramiento de los indicadores macroeconómicos no se tradujeron en trabajo formal en el país y tampoco las políticas sociales llegaron a cubrir demandas de mayorías ciudadanas. Es claro que en este sistema capitalista por más redistribución que se implemente quedan y quedarán personas sin acceso a la ciudadanía. Desde ese lugar se plantearon dos opciones desde la CTEP: "conformarnos con subsistir como "ciudadanos de segunda" magramente asistidos por el estado en las periferias del mercado - construir una nueva economía que rompa con la lógica de la ganancia, la Economía Popular (E.P)" (CTEP).

19 Para Grabois (2016) se trata de un nuevo sujeto que emerge de un paradigma socioeconómico global dónde el trabajo asalariado estable ya no es más un el cursus honorum de la clase obrera y se consolida un nuevo segmento compuesto no por microempresarios, ni asistidos sino por trabajadores.

20 Es auspicioso el cambio paradigmático en cuanto a la transversalidad de la perspectiva de género(s) en el ámbito del Estado y su traducción en los presupuestos y políticas públicas. Tal es el caso de la jerarquización del Ministerio de las Mujeres, Géneros y Diversidad, programas: de aportes por tareas de cuidado, contra la violencia, promoción de oficios sin prejuicios entre otros.

21 El contexto de pandemia redujo los encuentros y eso genera "reclamos en las consultas y decisiones que toma la comisión" (M. criancera integrante de la cooperativa). 